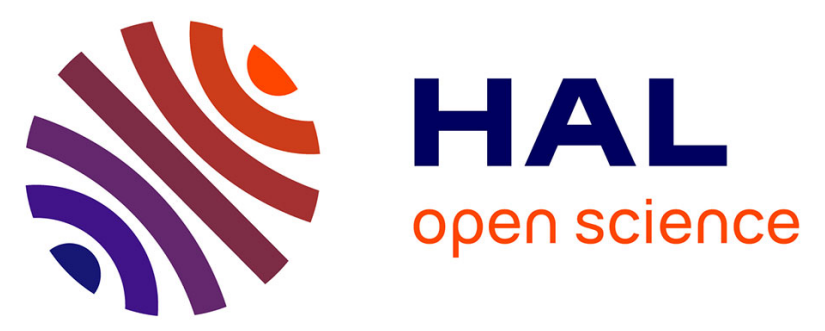

\title{
Value of assessment of multivoxel proton chemical shift imaging to predict long term outcome in patients after out-of-hospital cardiac arrest: A preliminary prospective observational study
}

Hervé Quintard, Lionel Velly, Salah Boussen, Xavier Chiosi, Marie-Eve Amoretti, Élodie Cervantes, Carole Ichai

\section{To cite this version:}

Hervé Quintard, Lionel Velly, Salah Boussen, Xavier Chiosi, Marie-Eve Amoretti, et al.. Value of assessment of multivoxel proton chemical shift imaging to predict long term outcome in patients after out-of-hospital cardiac arrest: A preliminary prospective observational study. Resuscitation, 2018, 134, pp. 136-144. 10.1016/j.resuscitation.2018.09.007 . hal-01980081

\section{HAL Id: hal-01980081 \\ https://hal.science/hal-01980081}

Submitted on 21 Oct 2021

HAL is a multi-disciplinary open access archive for the deposit and dissemination of scientific research documents, whether they are published or not. The documents may come from teaching and research institutions in France or abroad, or from public or private research centers.
L'archive ouverte pluridisciplinaire HAL, est destinée au dépôt et à la diffusion de documents scientifiques de niveau recherche, publiés ou non, émanant des établissements d'enseignement et de recherche français ou étrangers, des laboratoires publics ou privés.

\section{(ㄷ)(1) $\$$}

Distributed under a Creative Commons Attribution - NonCommerciall 4.0 International 


\section{Clinical paper}

2

\section{3}

\section{Value of Assessment of Multivoxel Proton Chemical Shift Imaging to} Predict Long Term Outcome in Patients after Out-of-hospital Cardiac Arrest: A Preliminary Prospective Observational Study.

Hervé Quintard ${ }^{\mathrm{a}, \mathrm{b}^{*}}$; Lionel Vellyc,d ; Salah Boussen ${ }^{\mathrm{c}, \mathrm{e}}$, Xavier Chiosia ${ }^{\mathrm{a}}$; Marie-Eve Amorettif; Elodie Cervantes ${ }^{\mathrm{f}}$; Carole Ichai ${ }^{\mathrm{a}}$

a Université Côte d'Azur, CHU de Nice, Department of Anaesthesiology and Critical Care Medicine, Hôpital Pasteur 2, Nice, France.

${ }^{\mathrm{b}}$ CNRS, UMR 7275, Sophia Antipolis, France.

c Aix Marseille University, Department of Anaesthesiology and Critical Care Medicine, University Hospital La Timone, Marseille, France.

${ }^{\mathrm{d}}$ Aix Marseille University, Institut de Neuroscience de la Timone (INT), Marseille, France.

e Aix Marseille University, Laboratoire de Biomécanique Appliquée (IFSTTAR), Marseille, France.

f Université Côte d'Azur, CHU de Nice, Radiology department, Hôpital Pasteur 2, Nice, France.

* Corresponding author: Dr Hervé QUINTARD, Service d'Anesthésie Réanimation, CHU de Nice, Hôpital Pasteur 2, 30 voie romaine 06000 NICE.

Tel : +33492033300; mail : quintard.h@chu-nice.fr

Word count abstract: 247

Word count manuscript: 3562

References: 39

Illustrations in main manuscript: 3 Tables, 3 Figures 
30

31

32

33

34

35

36

Illustrations in supplementary appendix: 3 additional files

KEYWORDS: Cardiac arrest, outcome, prognosis, magnetic resonance imaging, chemical shift imaging, magnetic resonance spectroscopic, hypoxic ischemic encephalopathy, diffusion weighted imaging 


\section{ABSTRACT}

Background : Existing methods to predict recovery after out-of-hospital cardiac arrest (OHCA) lack of accuracy. The aim of this study was to determine whether quantitative proton chemical shift imaging $\left({ }^{1} \mathrm{H}-\mathrm{CSI}\right)$ during the subacute stage of $\mathrm{OHCA}$ can predict neurological outcome of such patients.

Methods : This monocentric prospective observational study was conducted in a Intensive Care Unit of a teaching hospital. Forty consecutive patients with OHCA were enrolled between January 1st 2011 to December 31st 2013. Multivoxel ${ }^{1} \mathrm{H}$-CSI values were compared to structural magnetic resonance imaging (MRI) sequences (fluid-attenuated inversion recovery and diffusion-weighted imaging). Ratios of $\mathrm{N}$-acetyl-aspartate (NAA) to creatine ( $\mathrm{Cr}$ ) and choline compounds were analyzed using region of interest in bilateral lenticular cores and thalami. The outcome evaluated was the Cerebral Performance Category (CPC) at 6 months, dichotomized as favorable (CPC1-2) and unfavorable outcome (CPC3-5). The performance was compared by area under the receiver operating characteristic ( $\mathrm{ROC}_{\mathrm{AUC}}$ ) curves analysis.

Results : Twenty nine OHCA had an interpretable MRI. Eight patients (28\%) had favorable outcome at 6 months. The worst NAA/Cr in lenticular cores was the best ${ }^{1} \mathrm{H}$-CSI marker, with $80 \%$ sensitivity ( $95 \%$ confidence interval $(\mathrm{Cl}), 57-94)$ and a $100 \%$ specificity $(95 \% \mathrm{Cl}, 63-100)$ with a positive predictive value of $100 \%$. Prognostic accuracy, as quantified by the ROC $A \cup$, was higher with the worst $\mathrm{NAA} / \mathrm{Cr}$ in lenticular cores $\left(\mathrm{ROC}_{\mathrm{AUC}} 0.88 ; 95 \% \mathrm{Cl}, 0.70-0.97\right)$ than with the structural MRI sequences.

Conclusion : In this preliminary study we found that multivoxe ${ }^{1} \mathrm{H}$-CSI in lenticular cores, was highly predictive of unfavorable outcome at 6 months. 


\section{INTRODUCTION}

Out-of-hospital cardiac arrest (OHCA) is a major cause of death and disability with an annual incidence of 60 for 100,000 habitants [1]. Numerous efforts have been made to develop tools that help in detecting injury severity and subsequent outcomes. The more-commonly used indicators of OHCA severity include the initial cardiac rhythm, the length of resuscitation, the initial Glasgow Coma Scale, and the presence of nonreactive pupils [2]. However the clinical course of neurological recovery is obscured by sedation and hypothermia [3]. An early assessment of unfavorable prognosis based on the sole clinical examination may contribute to withdrawal of life sustaining therapy in nearly $20 \%$ of patients who may otherwise have shown complete neurological recovery [2]. The development of additional prediction tools, including electrophysiological examinations (electroencephalography and somatosensory evoked potentials), neuroimaging and chemical biomarkers as protein $\mathrm{S} 100 \beta$ or Neuron Specific Enolase may help to evaluate the extent of brain injury [3]. Considering the limits of sensitivity and specificity of these predictive tools, last published guidelines on OHCA recommend delaying prognostication after therapeutic hypothermia and using multiple prognostic tools [4-6].

Advances in MRI-based neuroimaging techniques, as diffusion tensor imaging, magnetisation transfer ratio, and perfusion imaging offer new potential to brain injury prognosis [7]. Multivoxel in vivo proton chemical shift imaging $\left({ }^{1} \mathrm{H}-\mathrm{CSI}\right)$ known as magnetic resonance spectroscopic (MRS) imaging has also the advantage of being a unique noninvasive method to quantify the magnitude and regional distribution of injury [8]. ${ }^{1} \mathrm{H}-\mathrm{CSI}$ enables to measure levels of several metabolites markers of neuronal integrity and cell membrane turnover [9]. $\mathrm{N}$-acetylaspartate (NAA) levels are commonly used as a marker for neuronal loss after 

5

traumatic or hypoxic-ischemic brain injury, Creatinin and phosphocreatine $(\mathrm{Cr})$ resonance levels may represent gliosis, Choline (Cho) resonance represents cell membrane turnover [8]. Althought several studies have been conducted using this technique in TBI patients with inconsistent results [10], few data are available for post ischemic patients, especially following OHCA. Most of the studies have been conducted in perinatal hypoxia preterm or term newborn with consistent results on the prognosis value of the NAA/Cr ratio mesured in striatum [11-13]. In adults patients after OHCA, limited data on ${ }^{1} \mathrm{H}-\mathrm{CSI}$ have been reported to assess prognosis [14-16].

The goal of this study was to assess and validate the prognostic performance of ${ }^{1} \mathrm{H}-\mathrm{CSI}$ in the subacute phase (7 to 14 days post insult) of hypoxic-ischemic brain injury in OHCA patients. 


\section{MATERIALS AND METHODS}

100

101

102

103

104

\section{Study population}

All comatose patients (Glasgow Coma Scale, GCS < 9) on admission aged 18 to 80 years admitted from January 1st 2011 to December 31st 2013, for OHCA from cardiac or respiratory causes were eligible. Pregnant women, and patient with contraindication to magnetic exposure were excluded from the study. The study protocol was approved by the local institutional ethics committee (Comité de Protection des Personnes Sud Méditerrranée V, study no. 2011-A00683-38), written informed consent was obtained from the next-of-kin before enrolment. When possible, patient's consent was obtained in case of favorable neurologic outcome in accordance with the French law.

\section{Patient management}

Patients were treated according to a standardised institutional protocol that complied with the International Liaison Committee On Resuscitation recommendations [6] and has been described elsewhere [17]. Briefly, after return of spontaneous circulation obtained by the medical prehospital team, coronary angiography and percutaneous coronary intervention were performed if needed. Then patients were admitted in our 40 beds intensive care unit (ICU) of our tertiary care university hospital. All comatose OHCA patients were treated with a target body temperature for $24 \mathrm{~h}$ according to guidelines, were sedated with an association of midazolam and fentanyl during hypothermia and paralyzed using continuous infusion of cisatracurium. All patients were intubated and mechanically ventilated aiming at a $\mathrm{PaO}_{2}$ between 75 and $100 \mathrm{mmHg}$ and $\mathrm{PaCO}_{2}$ between 35 and $45 \mathrm{mmHg}$. A central venous catheter and an arterial line were inserted for monitoring respectively central venous and arterial blood pressure, and blood sampling. Therapeutic goals were a mean arterial 
122

123

124

125

pressure $>80 \mathrm{mmHg}$ and urine output $>0.5 \mathrm{~mL} / \mathrm{kg} / \mathrm{hr}$. These goals were reached by giving fluid infusion or dobutamine or norepinephrine according to hemodynamic monitoring data.

Glucose was maintained between 0.8 and $1.2 \mathrm{~g} / \mathrm{dL}$ by continuous insulin infusion according to a local protocol.

\section{Data collection}

Demographic and clinical informations were collected prospectively with an Utstein-style data [18]. Glasgow Coma Scale [19] was assessed at hospital admission, and day of MRI acquisition and arterial blood lactate levels on admission and at 24 hours later, OHCA score [20] was computed for each patient (Supplemental, additional file 1). The severity of disease was characterized by the Simplified Acute Physiology Score II [21] on the first day after admission.

\section{MR Imaging Data Acquisition}

MR imaging was performed mean 7 days after OHCA, according to tolerance of transport of such patients. Structural MRI and ${ }^{1} \mathrm{H}-\mathrm{MRS}$, were performed on 1.5-T Signa HDxt imager (GE Healthcare, Milwaukee, WI, USA). Pulse sequences included a T2-weighted sequence (axial; slice thickness, $5 \mathrm{~mm}$; TR/TE, 6,400/105 ms; bandwidth, $122 \mathrm{~Hz}$; flip angle, 90; matrix, 512x320; field of view (FOV), $240 \mathrm{~mm}$ ), T2*-weighted sequence (axial; slice thickness, $5 \mathrm{~mm}$; TR/TE, 640/18 ms; bandwidth, $57 \mathrm{~Hz}$; flip angle, $20^{\circ}$; matrix, 352x224; FOV, $250 \mathrm{~mm}$ ), fluidattenuated inversion recovery (FLAIR; axial; slice thickness, 5 mm; TR/TE/TI, 9,000/160/2,250 ms; bandwidth, $122 \mathrm{~Hz}$; flip angle, 90; matrix, 256x192; FOV, $250 \mathrm{~mm}$ ), and diffusionweighted imaging (DWI; axial; slice thickness, $3 \mathrm{~mm}$; TR/TE, 6,150/102 ms; max b value of 1,000 s/mm2; bandwidth, $1953 \mathrm{~Hz}$; flip angle, 90; matrix, 128x128; FOV, $250 \mathrm{~mm}$ ). Structural MRI acquisition took about 20 min, depending on the number of slices required to 
cover the brain. Multivoxel ${ }^{1} \mathrm{H}-\mathrm{CSI}$ was acquired using the point-resolved proton spectroscopy sequence (PRESS) with the following parameters: slice thickness, $20 \mathrm{~mm}$; TR/TE, 1000/144 ms; matrix, 16x16; FOV, 240 mm; number of excitation, 1 . The axial ${ }^{1} \mathrm{H}-\mathrm{MRS}$ covering a region from the head of striatum to the thalamus. This acquisition took $11 \mathrm{~min}$. The MR images were retrieved in DICOM (Digital Imaging and Communications in Medicine) format from picture archiving and communication system servers at the hospitals.

\section{Image Processing}

Brain MR images were reviewed by a board-certified blinded radiologist (ME.A, with 10 years of postfellowship experience) to evaluate possible structural abnormalities. Participants with any evidence of territorial stroke of any age, intracranial hemorrhage, or intracranial mass lesions were excluded; in addition, any subject with head motion greater than $2 \mathrm{~mm}$ across the imaging session was excluded. MR imaging morphologic analysis was accomplished using a validated score on the basis of a visual rating. In the FLAIR-DWI scoring (Supplemental, additional file 2) proposed by Hirsch et al., [22] developed in a similar population with OHCA, the anatomic location and degree of signal abnormality on FLAIR images and DWI sequences is rated from 0 (normal) to 4 (severe), and composite tissue signal change is then calculated in cortical, basal ganglia and thalamus, brainstem, and cerebellar structures. The sum of scores in cortical, basal ganglia, and all structures is referred to as the "cortex", "deep gray nuclei", and "overall score", respectively. The sum or all scores recorded by using FLAIR and DWI is known as the DWI-FLAIR score, respectively [22].

Multivoxel ${ }^{1} \mathrm{H}-\mathrm{CSI}$ data processing was performed by an expert radiologist allowing to measure the area under the curve by standard manufacturer software dedicated to MR spectroscopy post-processing (Advantage Windows for General Electric). The volume of interest was placed carefully to avoid contact with cerebral spinal fluid on non-angled FLAIR 
images after coregistration of spectroscopic data and FLAIR volume (Figure 1). The spectra were analyzed to determine the concentration of metabolites in the lenticular cores (right and left side) and thalami (right and left side): NAA (at $2.0 \mathrm{ppm}$ ), Cho (at $3.2 \mathrm{ppm}$ ) and $\mathrm{Cr}$ (at $3.0 \mathrm{ppm})$. The quality of the selected spectra was visually inspected and was considered acceptable only if Cho and $\mathrm{Cr}$ signals were clearly separated. The spectra were also rejected if MR spectroscopists detected artefacts such as large baseline distortions, exceptionally broadened metabolite peaks, insufficient removal of the water line, large phase errors, and signals originating from outside the voxel as conventionally used in clinical practice. In all cases, peak surface area was used to calculate metabolite ratios for metabolite quantification. The worst value of bilateral lenticular cores and thalami were analysed except in cases of some non interpretable voxels. In this case, the sole side with spectra of good quality was taken into account.

\section{Outcome}

Physicians in charge of the patients were blinded to ${ }^{1} \mathrm{H}$-CSI but had access to conventional MRI sequences (without FLAIR-DWI scoring) as well as all clinical, biological, and electrophysiological data. All clinical decisions remained at the discretion of the treating team.

Neurologic performance was assessed at 6 months according to Glasgow-Pittsburgh Cerebral Performance Categories (CPC; Supplemental, additional file 3) [23] based on data issued from medical files or from telephone calls. Briefly, CPC 1 as normal, CPC 2 as moderate disability, CPC 3 as severe disability, CPC 4 as persistent vegetative state, and CPC 5 as death. CPC at 6 months was the primary outcome measure for stratifying patients into favorable outcomes (CPC 1-2) and unfavorable outcome (CPC 3-5). 


\section{Statistical analysis}

Data were tested for normality and are presented as mean \pm standard deviation or median [interquartile range (IQR)] for continuous variables when appropriate. Categorical variables are presented as $\mathrm{n}(\%)$. Comparisons between groups according to outcome at 6 months were performed using Mann-Whitney $U$ test or $\chi 2$ test when appropriate. Univariate logistic regression models were used to examine the predictive value of each variable. Additional details of odds ratios are available in the appendix (Supplemental). For each predictive variable $(\mathrm{P}<0.05)$, a receiver-operating-characteristic curve was plotted, and the corresponding area under the curve (ROC AUC) was computed. An optimal cutoff was defined as the value leading to the maximal sensitivity at $100 \%$ specificity. For this optimal cutoff, specificity, sensitivity, and positive and negative predictive values were computed, with 95\% Confidence Interval $(\mathrm{Cl})$ computed by bootstrap. The ROC $\mathrm{AUC}_{\mathrm{C}}$ and sensitivity of each variable were compared using the method described by DeLong et al. [24] and Fisher's exact test, respectively. All these statistics were also computed for three multivariate logistic regression models. Multivariate analysis was performed using backward stepwise logistic regression to enter variables that yielded $P$ values smaller than $<0.1$ in the univariate analysis to identify

210 factors that independently predicted the 6 months outcome $P<0.05$ was considered 211 statistically significant. Statistical analysis was performed using MATLAB R2017a and 212 Statistics and Machine Learning Toolbox 11.1 (The MathWorks Inc., Natick, MA). 


\section{RESULTS}

215

During the selected period, 40 patients admitted in our intensive care unit after a OHCA met the inclusion criteria, 29 were included in the analysis with an interpretable multi-modal MRI (see consort diagram Figure 2); 8 (28\%) had a favorable neurologic outcome (5 with a CPC score of 1 and 3 with a CPC of 2). Unfavorable outcome was reported in 21 patients: 17 (81\%) died during hospital stay with a median survival time of 16 days (IQR 12 to 40 days), at 6 months; one patient had CPC 4 and 20 CPC 5 (see Data in Brief, Table 1). Withdrawal or limitation-of-care decisions were taken for 15 patients (52\%). Characteristics of patients are summarized in Table 1. At baseline, there was no significant difference between patients with favorable or unfavorable outcome in terms of proportion of shockable rhythm or time to return of spontaneous circulation. Patients with a favorable outcome were younger $(P=0.03)$ and had a lower serum lactate level at $\mathrm{H} 24(P=0.04)$.

Median time to MRI was not significantly different in unfavorable- and favorable-outcome patients (7 (IQR 7 to 9 days) vs. 8 (IQR 6 to 12 days) days; P=0.66). The GCS was significantly lower in the unfavorable group compared to favorable one on the MRI-day (10 (IQR 9 to 12) vs. 3 (IQR 3 to 6); $p=0.005$; Table 2). FLAIR-DWI deep nuclei scores were significantly higher (i.e. worse) in the unfavorable outcome group (2 (IQR 0 to 13) vs. 1 (IQR 0 to 2); $p=0.05$; Table 2). FLAIR or DWI images showed mild-to-severe signal abnormalities in cortex in 4 patients with a favorable outcome.

In total, $11(27 \%){ }^{1} \mathrm{H}-\mathrm{CSI}$ spectra were not interpretable. The worst $\mathrm{NAA} / \mathrm{Cr}$ ratios in lenticular cores was significantly lower in the unfavorable- vs. favorable-outcome patients (1.12 \pm 0.37 vs. $1.62 \pm 0.34$ respectively; $P=0.002$; see Table 2$)$. There was no significant difference between group in $\mathrm{Naa} / \mathrm{Cr}$ ratios in the thalamus $(1.75 \pm 0.45$ vs. $1.71 \pm 0.64$ 
respectively; $P=0.87$; see Table 2 ). The NAA/Cho ratio measured in lenticular cores and thalami was not significantly different between groups (respectively $P=0.08$ and 0.67 ; see Table 2).

Figure 3 summarizes the results of ROC curves for the principal predictors of unfavorable outcome. Table 3 shows the cutoff value predictive of unfavorable outcome with a specificity of $100 \%$, the sensitivity value corresponding to this cutoff value and $\mathrm{ROC}_{\mathrm{AUC}}$ for each predictor. The optimal cutoff value of worst NAA/Cr in lenticulair cores was $<1.32$ with a specificity of $100 \%$ (sensitivity $80 \%$ ). The same cutoff value, similar specificity and sensitivity were obtained in adjusted analyses of the subpopulation excluding patients with withdrawal or limitation of care (see Data in Brief article Table 3).

Multivariate analyses and specific results are shown in Data in Brief article (Data in Brief article Table 2 and Figure 1). Briefly, in the first model, significant selected variables were SAPS II and Glasgow Coma Scale at MRI. The ROC AUC was 0.85 ( $95 \% \mathrm{Cl}, 0.71$ to 0.99 ). The second model, which included the FLAIR-DWI DNG score, increased the ROC AUC $_{\text {to }} 0.86$ (95\% $\mathrm{Cl}, 0.73$ to 0.99$)$. Finally, the third model, which included worst NAA/Cr in lenticular cores, increased the ROC $A \cup C$ to $0.95(95 \% \mathrm{Cl}, 0.87$ to 1.00$)$, with a specificity of $100 \%(95 \% \mathrm{Cl}, 63 \%$ to $100 \%)$ and a sensitivity of $90 \%(95 \% \mathrm{Cl}, 70 \%$ to $99 \%)$. 


\section{DISCUSSION}

260

261

262

263

The major findings of this prospective preliminary study sugget as follow: 1) The DWI-FLAIR "deep gray nuclei" score could be predictive prognostic factor in patient 7 days after OHCA. 2) The Worst NAA/Cr ratio measured in lenticular cores seems to be a feasible predictive factor also in this setting. 3) The association of clinical data (SAPS II and GCS at MRI time), and MRI data as the "deep gray nuclei" score and NAA/Cr ratio measured in lenticular core seems to be an accurate prognostic factor for patient after CA associating an excellent sensitivity (90\%) for a specificity of $100 \%$.

Anoxic or hypoxic- ischemic-encephalopathy (HIE) is difficult to predict on clinical grounds. Few studies recorded both MRI findings and long-term outcome [5]. Diffuse cortical abnormality by DWI in the acute phase following OHCA appears to be of unfavorable prognostic significance [25]. The Hirsh score has been described recently as an accurate scoring after cardiac arrest [22]. Using DWI and FLAIR sequences, Hirsch et al. [22] developed a score according to the importance of lesion in different cerebral areas. They reported a specificity of $100 \%$ and a sensitivity $50-60 \%$. Our data show a sensitivity of $57 \%$ with a specificity of $100 \%$ for a threshold of 8 in "deep gray nuclei" score. Such variation in accuracy could be explained by the delayed MRI exam in our study compared to Hirsch et al. one in which the median time to MRI was early, between $72-96 \mathrm{~h}$ after cardiac arrest. In our study, the median time to MRI was later, approximately 7 days corresponding to a subacute phase after injury. Indeed, an evolution of MRI brain variables over time has been recently reported in a newborn population after hypoxic lesion, especially within the first $72 \mathrm{~h}$ after injury [26]. Greer et al., [27] using serial MRI after adult cardiac arrest showed that in patient with unfavorable outcome there was a worsening in the signal abnormalities between the early phase (days 1-5) and the subacute period (days 6-12) with substantial new white 
matter changes on imaging, more often seen on DWI (100\%) than FLAIR (60\%). We decided to propose MRI exam 7 days after OHCA because in clinical practice, most discussions on therapeutic limitation take place during this period. Moreover, OHCA patients are commonly critical care patient with haemodynamic and respiratory instability, and MRI exam could be difficult at the early time following OHCA. Although Hirsh et al. described several patients with impossibility to undergo MRI exam because of agitation or haemodynamic instability, we report only 4 patients with movement artefact in our group.

The presence of the amino acid derivative $\mathrm{N}$-acetylaspartate (NAA) is almost exclusive to neurons and their processes and is, therefore, an accepted marker of their health [28-30] and, as such, its concentration will decrease with increasing damage to the brain[31]. We can also suggest that the 7 days delay for monitoring NAA concentration, a specific marker of neuronal damage, support the possibility of brain lesion detection. ${ }^{1} \mathrm{H}-\mathrm{CSI}$ was found to correlate with outcome of traumatic brain injury $[10,32]$ but has not been already well studied in adults after OHCA. In neonatal $\mathrm{HIE},{ }^{1} \mathrm{H}-\mathrm{CSI}$ of the basal ganglia and thalamus has been used to predict an adverse outcome [33]. Boichot et al. described a decrease in NAA/Cr ratio in severe neonatal HIE with unfavorable prognosis [12]. This decrease seems not to be influenced by hypothermia as reported later [13]. In a recent meta-analysis, ${ }^{1} \mathrm{H}$-CSI was considered as the most accurate quantitative MRI biomarker within the neonatal period for prediction of neurodevelopmental outcome after HIE [34]. In adults, very few data are available, mostly case reports using the single voxel spectroscopy in central white matter [14-16]. Wartenberg et al. described the case of a OHCA patient who was investigated 7 days after injury with encouraging data of ${ }^{1} \mathrm{H}$-CSI compared to other prognostic assessment techniques such as electroencephalography, or clinical evaluation [35]. To our knowledge, our study is the first to assess ${ }^{1} \mathrm{H}$-CSI of lenticular cores and thalamus in a prospective cohort 
of OHCA. In our study, the clinically relevant values of NAA/Cr ratio is 1.32 , a threshold below

308 which the probability of unfavorable outcome is close to 100.

309

310

311

312

313

Our findings should be interpreted in light of the following potential limitations. First, we decided to perform ${ }^{1} \mathrm{H}$-CSI more than 6 days after OHCA. Most case reports studies have been conducted in the acute phase after CA (24-48h after CA) and focused on cerebral lactate measurement $[14,16]$. It is likely that metabolite concentration in the brain is likely to change over time after HIE and lactate doublet peak is likely to disappear as reported in newborns [26]. However, delaying MRI examination has several advantages. It allows a better hemodynamic and respiratory stabilization of the patients, improving patient safety during transport and MRI, an important concern for an exam without short-term benefit. In addition, the data are obtained after the period of rapidly changing neurodegenerative changes, improving the consistency of the data. Delayed ${ }^{1} \mathrm{H}$-CSI has been used to predict prognosis after brain trauma with a good sensitivity when MRI was performed 24 days after the injury [36]. Finally, patient recovery is often delayed for a few days due to the practice of hypothermia and sedation. Patients often regain consciousness between day 2 and day 5 and early ${ }^{1} \mathrm{H}$-CSI would lead to unnecessary studies. In our study, the mean Glasgow score in good recovery group at the MRI time, at 7 days, was only 10 . Despite allowing several days for stabilization in the ICU, we could obtain interpretable ${ }^{1} \mathrm{H}-\mathrm{CSI}$ in only $73 \%$ of our patients. This can probably be improved with increasing experience. Second, as in all others studies using MRS which is limited to the selected region of interest represents also a limitation. This point must be underlined because the neurochemical profile was reported to be variable across brain regions after an experimental focal ischemic lesion [37]. We chose to perform ${ }^{1} \mathrm{H}$-CSI measurements in 2 different regions of the brain (Lenticular core and thalamus) in order to limit the bias related to sampling choice. In fact, ${ }^{1} \mathrm{H}-\mathrm{CSI}$ in the thalami did not 
improve prognosis accuracy. We chose these 2 regions because they are easy to recognize and less prone to errors due to inaccurate positioning of voxel samples. They were also associated with prognosis in trauma patients [38] but the metabolic pathways of brain injury after brain trauma and OHCA are not similar. Neostriatal structures (putamen and caudate nucleus) are particularly sensitive to ischemia compared to globus pallidus or thalamus [39]. Thus, it is not surprising to find that ${ }^{1} \mathrm{H}-\mathrm{CSI}$ in the lenticular cores was associated with the best prognosis value in our study. Third, our methodological choices for ${ }^{1} \mathrm{H}-\mathrm{CSI}$ can be discussed. We used $\mathrm{Cr}$ as the reference metabolite concentration because it is the most stable one across patients. Although its concentration may vary in the early period after OHCA and hypothermia, probably delayed imaging limits the amplitude of this change between patients. Because of our short-time of echo, we were not able to study lactate ratio, which has been used for assessment of prognosis in previous studies. We chose to limit the duration of MRI scanning for safety reasons but it could be interesting to evaluate brain lactate changes after OHCA. Finally, we cannot exclude misclassification of outcome due to "self-fulfilling" prophecies arising from premature decisions of withdrawal and limitation of life-sustaining treatments. However, even if we exclude all patients who died from care withdrawal, the cutoff for NAA/Cr ratios measured in lenticular cores and AUC did not vary significantly (Table 3 Data in Brief).

In conclusion, according our results we suggest that in adult patients after cardiac arrest that

${ }^{1} \mathrm{H}$-CSI measured on lenticular cores at the subacute phase of hypoxic-ischemic brain injury is feasible in most patients and may predict long-term recovery. This evidence requires statistical confirmation from future large-scale trials in association with other ancillary tests (EEG, SSEP, pupillometry...) with strict protocol of withdrawal or limitation-of-care decisions. 
355

Source of funding : This study was not supported by any funding.

Conflict of interest statement: The authors declare that they have no competing interests.

Author contributions: $\mathrm{HQ}$ was the lead intensivist and contributed to the protocol development, study design and concept, conduct, data acquisition and coordination, data interpretation, writing and revising it critically, and reviewing the report. He takes responsibility for the integrity of the data and the accuracy of the data analysis. LV was involved in data interpretation, writing and reviewing the report. SB was involved in data analysis, contribution to the statistical analysis plan, statistical analysis. XC was involved in data collection and preparation of the report. MEA, EC were involved in data acquisition, data analysis, and revising the report. $\mathrm{Cl}$ was involved in data interpretation, and revising the report critically. All authors approved the final version. 
[1] Stecker EC, Reinier K, Marijon E, Narayanan K, Teodorescu C, Uy-Evanado A, et al. Public Health Burden of Sudden Cardiac Death in the United States. Circ Arrhythm Electrophysiol 2014;7:212-7.

[2] Perman SM, Kirkpatrick JN, Reitsma AM, Gaieski DF, Lau B, Smith TM, et al. Timing of neuroprognostication in postcardiac arrest therapeutic hypothermia*: Crit Care Med 2012;40:71924.

[3] Taccone FS, Cronberg T, Friberg H, Greer D, Horn J, Oddo M, et al. How to assess prognosis after cardiac arrest and therapeutic hypothermia. Crit Care 2014;18:202.

[4] Cronberg T, Brizzi M, Liedholm LJ, Rosén I, Rubertsson S, Rylander C, et al. Neurological prognostication after cardiac arrest-Recommendations from the Swedish Resuscitation Council. Resuscitation 2013;84:867-72.

[5] Sandroni C, Cariou A, Cavallaro F, Cronberg T, Friberg H, Hoedemaekers C, et al. Prognostication in comatose survivors of cardiac arrest: An advisory statement from the European Resuscitation Council and the European Society of Intensive Care Medicine. Resuscitation 2014;85:1779-89.

[6] Nolan JP, Soar J, Cariou A, Cronberg T, Moulaert VRM, Deakin CD, et al. European Resuscitation Council and European Society of Intensive Care Medicine Guidelines for Post-resuscitation Care 2015. Resuscitation 2015;95:202-22.

[7] Shenton ME, Hamoda HM, Schneiderman JS, Bouix S, Pasternak O, Rathi Y, et al. A review of magnetic resonance imaging and diffusion tensor imaging findings in mild traumatic brain injury. Brain Imaging Behav 2012;6:137-92.

[8] Castillo M, Kwock L, Mukherji SK. Clinical applications of proton MR spectroscopy. Am J Neuroradiol 1996;17:1-15.

[9] Moffett J, Ross B, Arun P, Madhavarao C, Namboodiri A. N-Acetylaspartate in the CNS: From neurodiagnostics to neurobiology. Prog Neurobiol 2007;81:89-131.

[10] Croall I, Smith FE, Blamire AM. Magnetic resonance spectroscopy for traumatic brain injury. Top Magn Reson Imaging 2015;24:267-274.

[11] Zarifi MK, Astrakas LG, Poussaint TY, Plessis A du, Zurakowski D, Tzika AA. Prediction of Adverse Outcome with Cerebral Lactate Level and Apparent Diffusion Coefficient in Infants with Perinatal Asphyxia. Radiology 2002;225:859-70.

[12] Boichot C, Walker PM, Durand C, Grimaldi M, Chapuis S, Brunotte F. Term neonate prognoses after perinatal asphyxia: contributions of MR imaging, MR spectroscopy, relaxation times, and apparent diffusion coefficients. Radiology 2006;239:839-848. MR spectroscopy and diffusion tensor imaging in newborns with hypoxic-ischemic encephalopathy treated by brain cooling. Neuroradiology 2013;55:1017-25.

407 [14] Martin GB, Paradis NA, Helpern JA, Nowak RM, Welch KMA. Nuclear magnetic resonance 408 spectroscopy study of human brain after cardiac resuscitation. Stroke 1991;22:462-468. 
[15] Berek K, Lechleitner P, Luef G, Felber S, Saltuari L, Schinnerl A, et al. Early determination of neurological outcome after prehospital cardiopulmonary resuscitation. Stroke 1995;26:543-9.

[16] Kano H, Houkin K, Harada K, Koyanagi I, Nara S, Itou Y, et al. Neuronal cell injury in patients after cardiopulmonary resuscitation: evaluation by diffusion-weighted imaging and magnetic resonance spectroscopy. Neurosurg Rev 2006;29:88-92.

[17] Orban J-C, Novain M, Cattet F, Plattier R, Nefzaoui M, Hyvernat H, et al. Association of serum lactate with outcome after out-of-hospital cardiac arrest treated with therapeutic hypothermia. PLOS ONE 2017;12:e0173239.

[18] Jacobs I, Nadkarni V, Bahr J, Berg RA, Billi JE, Bossaert L, et al. Cardiac arrest and cardiopulmonary resuscitation outcome reports: update and simplification of the Utstein templates for resuscitation registries. Resuscitation 2004;63:233-49.

[19] Teasdale G, Gentleman D. The Description of 'Conscious Level': A Case for the Glasgow Coma Scale. Scott Med J 1982;27:7-9.

[20] Adrie C, Cariou A, Mourvillier B, Laurent I, Dabbane H, Hantala F, et al. Predicting survival with good neurological recovery at hospital admission after successful resuscitation of out-of-hospital cardiac arrest: the OHCA score. Eur Heart J 2006;27:2840-5.

[21] Le Gall JR, Lemeshow S, Saulnier F. A new Simplified Acute Physiology Score (SAPS II) based on a European/North American multicenter study. JAMA 1993;270:2957-63.

[22] Hirsch KG, Mlynash M, Jansen S, Persoon S, Eyngorn I, Krasnokutsky MV, et al. Prognostic Value of A Qualitative Brain MRI Scoring System After Cardiac Arrest: Prognostic value of qualitative MRI after cardiac arrest. J Neuroimaging 2015;25:430-7.

[23] Brain Resuscitation Clinical Trial I Study Group*. Randomized Clinical Study of Thiopental Loading in Comatose Survivors of Cardiac Arrest. N Engl J Med 1986;314:397-403.

[24] DeLong ER, DeLong DM, Clarke-Pearson DL. Comparing the areas under two or more correlated receiver operating characteristic curves: a nonparametric approach. Biometrics 1988;44:837-45.

[25] Choi SP, Park KN, Park HK, Kim JY, Youn CS, Ahn KJ, et al. Diffusion-weighted magnetic resonance imaging for predicting the clinical outcome of comatose survivors after cardiac arrest: a cohort study. Crit Care 2010;14:R17.

[26] Gano D, Chau V, Poskitt KJ, Hill A, Roland E, Brant R, et al. Evolution of pattern of injury and quantitative MRI on days 1 and 3 in term newborns with hypoxic-ischemic encephalopathy. Pediatr Res 2013;74:82-7.

[27] Greer D, Scripko P, Bartscher J, Sims J, Camargo E, Singhal A, et al. Serial MRI changes in comatose cardiac arrest patients. Neurocrit Care 2011;14:61-7.

[28] Simmons ML, Frondoza CG, Coyle JT. Immunocytochemical localization of N-acetyl-aspartate with monoclonal antibodies. Neuroscience 1991;45:37-45.

[29] Jenkins BG, Klivenyi P, Kustermann E, Andreassen OA, Ferrante RJ, Rosen BR, et al. Nonlinear decrease over time in $\mathrm{N}$-acetyl aspartate levels in the absence of neuronal loss and increases in glutamine and glucose in transgenic Huntington's disease mice. J Neurochem 2000;74:2108-19.

[30] Moffett JR, Ross B, Arun P, Madhavarao CN, Namboodiri AMA. N-Acetylaspartate in the CNS: from neurodiagnostics to neurobiology. Prog Neurobiol 2007;81:89-131. 
449 [31] Rigotti DJ, Inglese $\mathrm{M}$, Gonen O. Whole-brain N-acetylaspartate as a surrogate marker of 450 neuronal damage in diffuse neurologic disorders. AJNR Am J Neuroradiol 2007;28:1843-9.

451 [32] Weiss N, Galanaud D, Carpentier A, Naccache L, Puybasset L. Clinical review: prognostic value of 452 magnetic resonance imaging in acute brain injury and coma. Crit Care 2007;11:230.

453 [33] Peden CJ, Rutherford MA, Sargentoni J, Cox IJ, Bryant DJ, Dubowitz LM. Proton spectroscopy of 454 the neonatal brain following hypoxic-ischaemic injury. Dev Med Child Neurol 1993;35:502-10.

455 [34] Thayyil S, Chandrasekaran M, Taylor A, Bainbridge A, Cady EB, Chong WKK, et al. Cerebral 456 Magnetic Resonance Biomarkers in Neonatal Encephalopathy: A Meta-analysis. PEDIATRICS 457 2010;125:e382-95.

458 [35] Wartenberg KE, Patsalides A, Yepes MS. Is Magnetic Resonance Spectroscopy Superior to 459 Conventional Diagnostic Tools in Hypoxic-Ischemic Encephalopathy? J Neuroimaging 2004;14:180-6.

460 [36] Tollard E, Galanaud D, Perlbarg V, Sanchez-Pena P, Le Fur Y, Abdennour L, et al. Experience of 461 diffusion tensor imaging and $1 \mathrm{H}$ spectroscopy for outcome prediction in severe traumatic brain 462 injury: Preliminary results*: Crit Care Med 2009;37:1448-55.

463 [37] Alf MF, Lei H, Berthet C, Hirt L, Gruetter R, Mlynarik V. High-resolution spatial mapping of 464 changes in the neurochemical profile after focal ischemia in mice: HIGH-RESOLUTION MRSI AFTER 465 FOCAL ISCHEMIA. NMR Biomed 2012;25:247-54.

466 [38] Carpentier A, Galanaud D, Puybasset L, Muller J-C, Lescot T, Boch A-L, et al. Early Morphologic 467 and Spectroscopic Magnetic Resonance in Severe Traumatic Brain Injuries Can Detect "Invisible Brain 468 Stem Damage” and Predict “Vegetative States." J Neurotrauma 2006;23:674-85.

469 [39] Falini A, Barkovich AJ, Calabrese G, Origgi D, Triulzi F, Scotti G. Progressive brain failure after 470 diffuse hypoxic ischemic brain injury: a serial MR and proton MR spectroscopic study. Am J 471 Neuroradiol 1998;19:648-652. 


\section{LEGEND OF FIGURES}

Figure 1. Acquisition and Spectral Fitting of Multivoxel Proton Chemical Shift Imaging $\left({ }^{1} \mathrm{H}-\right.$ CSI). Solid green squares on axial FLAIR MRI represent the size of ${ }^{1} \mathrm{H}-\mathrm{CSI}$ voxel. Spectrum samples showing variation of spectra across the region of interest (1: right lenticular core; 2: right thalamus; 4: left thalamus 5: left lenticular core). MR spectrums of sample voxel show $\mathrm{N}$-acetyl-aspartate (NAA; $2.0 \mathrm{ppm})$, creatine plus phosphocreatine ( $\mathrm{Cr} ; 3.0 \mathrm{ppm})$, and choline compounds (Cho; $3.2 \mathrm{ppm}$ ), as well as contaminating lipids.

Figure 2. CONSORT flow chart of the cohort. Assessment, analysis population, and follow-up of the patients in the trial.

Figure 3. Receiver-Operating-Characteristic Curves for unfavorable outcome. DNG denotes Deep Gray Nuclei; DWI, Diffusion Weighted Imaging; FLAIR, Fluid-Attenuated Inversion Recovery; MRI, Magnetic Resonance Imaging; NAA/Cr, N-Acetyl Aspartate over Creatinine ratios. 

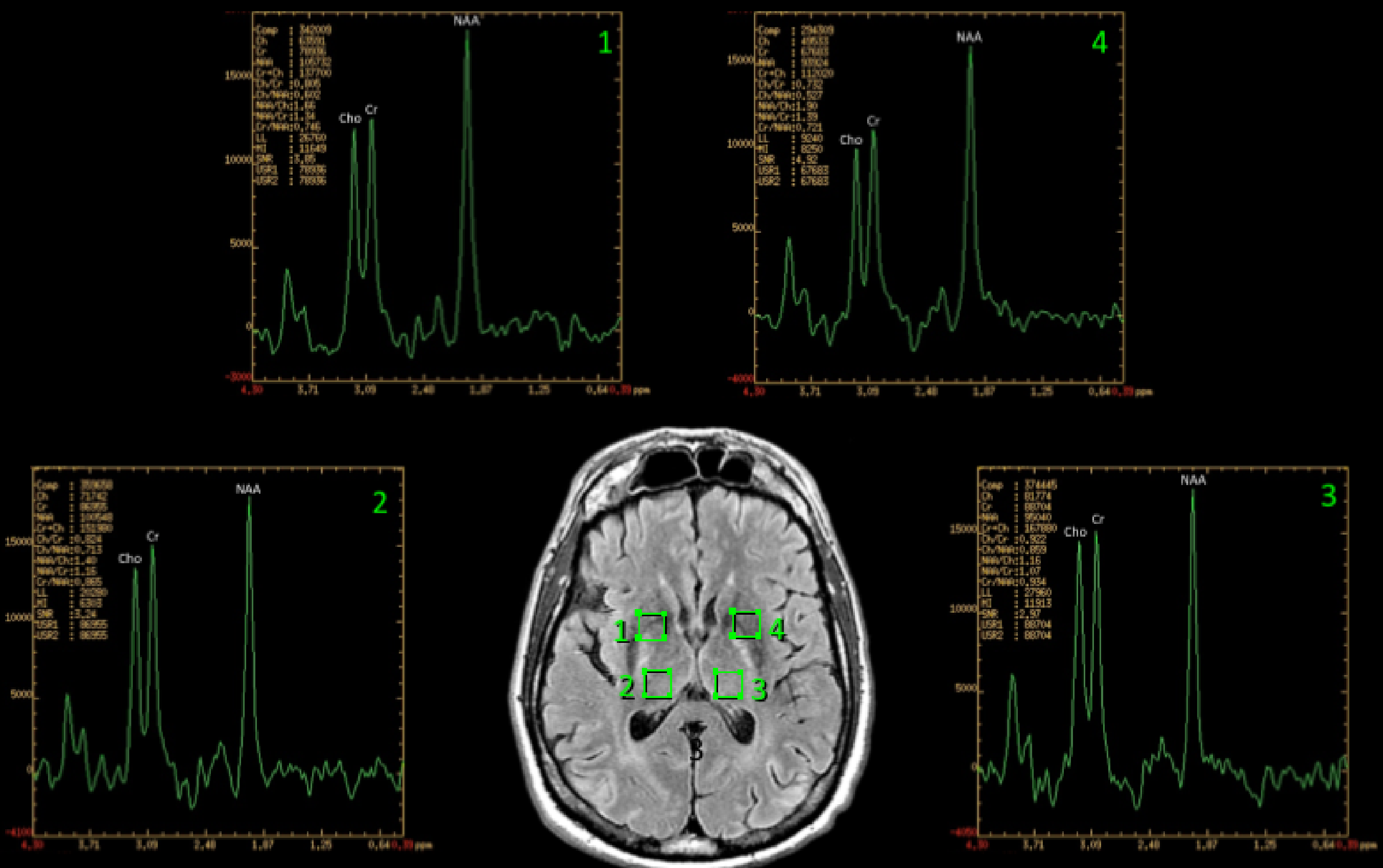
111 patients with cardiac arrest

\section{1 not included}

30 died before day 7 :

10 refractory multi-organ failure

8 new cardiac arrest

12 cerebral death

39 neurologic or traumatic cause of cardiac arrest 2 had MRI contraindication

40 included patients

\section{CSI were not interpretable}

4 movement artefacts

2 exam deletions

5 signal artefacts

29 analysed patients 


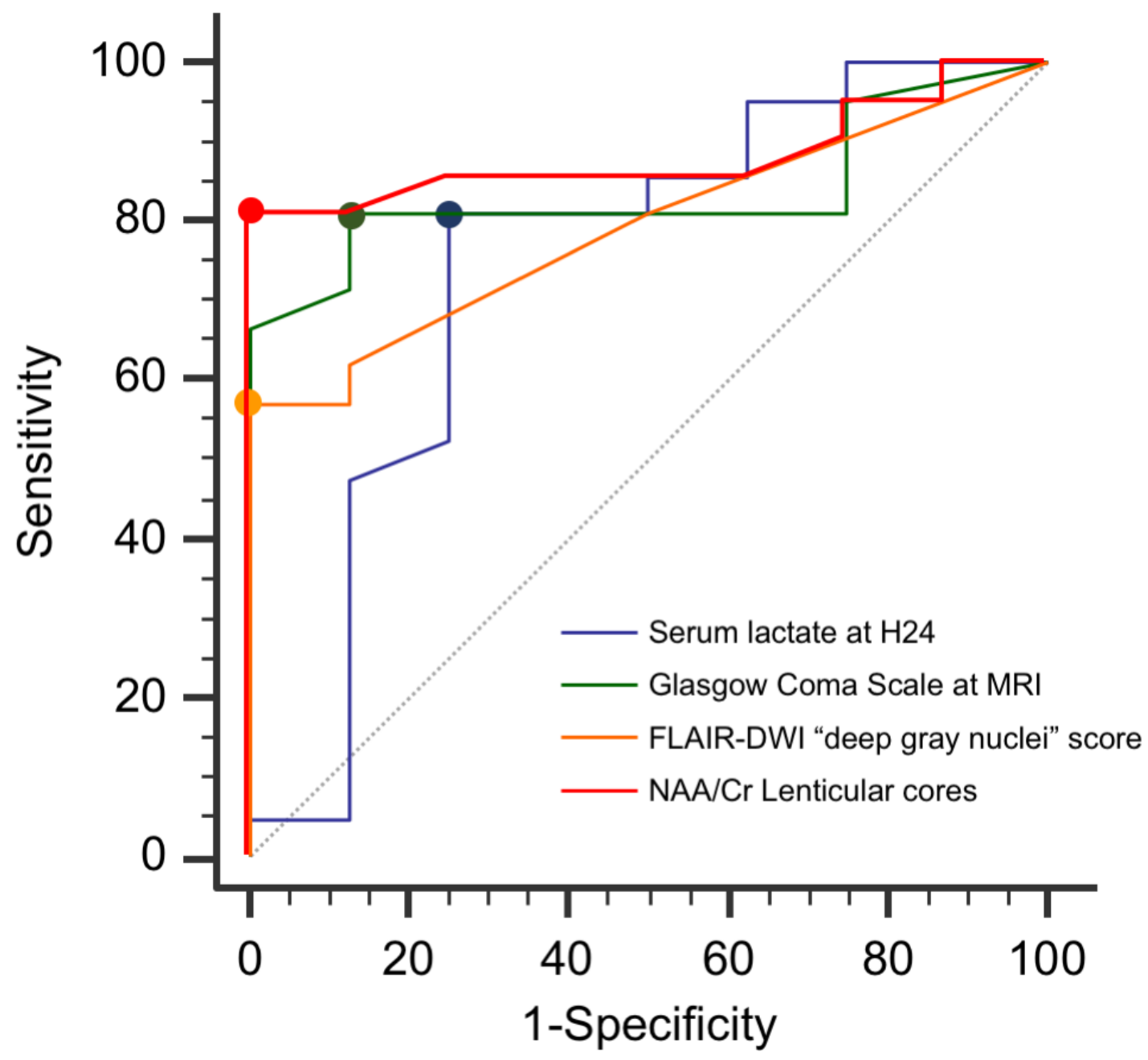


Table 1. Baseline Characteristics of the Patients.

\section{Characteristics}

Demographics

Age, years

Male sex, n (\%)

Arrest characteristics

Shockable rhythm, n (\%)

Ventricular fibrillation, $\mathrm{n}(\%)$

Non-perfusing ventricular tachycardia, n (\%)

Nonshockable, $\mathrm{n}(\%)$

Asystole, n (\%)

Pulseless electrical activity, $\mathrm{n}(\%)$

Unknown first rhythm, n (\%)

Epinephrine, n (\%)

Total dose of epinephrine during CPR, mg

Time to return of spontaneous circulation, $\mathrm{min}$

Postresuscitation

SAPS II

Corneal reflex absent, $\mathrm{n}(\%)$

Serum lactate on ICU admission, $\mathrm{mmol} / \mathrm{I}$

Serum creatinine on ICU admission, $\mu \mathrm{mol} / \mathrm{I}$

Percutaneous coronary artery intervention, $\mathrm{n}(\%)$

Hypothermia, n (\%)

Renal replacement therapy, n (\%)

Withdrawal or limitation of life sustaining therapy, $n$ (\%)

ICU mortality, n (\%)

ICU length of stay, days

Hospital mortality, n (\%)

$\begin{array}{ccc}\begin{array}{c}\text { All } \\ \text { patients }\end{array} & \begin{array}{c}\text { Favorable } \\ \text { outcome } \\ \text { (CPC 1-2) }\end{array} & \begin{array}{c}\text { Unfavorable } \\ \text { outcome } \\ (\mathrm{CPC} 3-5)\end{array} \\ (\mathrm{N}=29) & (\mathrm{N}=8) & (\mathrm{N}=21)\end{array}$

$58 \pm 14$

$50 \pm 14$

$62 \pm 12$

0.03

23 (79)

$6(75)$

$17(81)$

1.00

17 (59)

$6(75)$

$11(52)$

0.41

$12(41)$

$3(37)$

$9(43)$

1.00

$2(7)$

$1(13)$

1 (5)

0.48

$12(41) \quad 2(25)$

$10(48)$

0.41

$10(35)$

$2(25)$

$8(38)$

0.68

2 (7)

$0(0)$

2 (10)

1.00

$2(7)$

$2(25)$

$0(0)$

0.07

$21(72)$

$5(63)$

$16(76)$

0.65

3 [1-6]

1 [0-3]

4 [2-6]

0.12

18 [11-25]

16 [15-22]

19 [11-29]

0.92

11 (38)

$51 \pm 15$

$65 \pm 19$

0.07

$5(63)$

6 (29)

0.20

$3.2[1.5-6.4]$

$2.2[0.8-3.6]$

3.4 [1.7-6.5]

0.13

99 [81-132]

88 [80-97]

113 [81-138]

0.25

13 (45)

$5(62)$

$8(38)$

0.40

23 (82)

$6(75)$

17 (85)

1.00

8 (28)

2 (25)

6 (29)

1.00

15 (14)

0 (0)

15 (71)

$<0.001$

8 (28)

$0(0)$

8 (38)

0.003

13 [8-18]

11 [7-16]

14 [9-21]

0.33

9 (31)

$0(0)$

9 (43)

0.03

Data are expressed as median [IQR], means \pm SD or counts (\%) when appropriate.

CPC denotes Cerebral Performance Categories; CPR, cardiopulmonary resuscitation; ICU, intensive care unit; SAPS II, Simplified Acute Physiology Score II.

+ P-value for patients with a best numerical CPC 1 and 2 (favorable outcome) vs. those with categories 3 through 5 (unfavorable outcome). 
Table 2. Distribution of Variables in Prognostic after Cardiac Arrest.

\section{Variables}

Clinical and biological criteria in prognostication after cardiac arrest Serum lactate at $\mathrm{H} 24-\mathrm{mmol} / \mathrm{l}$

Glasgow Coma Scale at MR

OHCA score

Qualitative Magnetic Resonance Imaging (MRI) variables

FLAIR and DWI MRI interpretation - no. (\%)

$$
\text { Hyperintensity in deep gray nuclei }
$$

Hyperintensity in cortical areas

\section{FLAIR-DWI scoring system}

\section{Overall score}

Cortex score

Deep gray nuclei score

Quantitative MRI variables

${ }^{1} \mathrm{H}$-CSI N-acetyl aspartate over creatine ratio (NAA/Cr)

$$
\begin{aligned}
& \mathrm{NAA} / \mathrm{Cr} \text { Thalami } \\
& \mathrm{NAA} / \mathrm{Cr} \text { Lenticular cores }
\end{aligned}
$$

${ }^{1} \mathrm{H}$-CSI N-acetyl aspartate over Cho ratio (NAA/Cho)

$$
\text { NAA/Cho Thalami }
$$

NAA/Cho Lenticular cores

\begin{tabular}{cc} 
All patients & $\begin{array}{c}\text { Favorable } \\
\text { outcome } \\
(\text { CPC 1-2) }\end{array}$ \\
\hline$(N=29) \quad(N=8)$
\end{tabular}

\section{Unfavorable}

(CPC 3-5)

$(\mathrm{N}=21)$

P-valuet

$\begin{array}{cccc}1.2[1.0-2.2] & 1.0[0.6-1.1] & 1.5[1.1-2.3] & 0.04 \\ 5[3-10] & 10[9-12] & 3[3-6] & 0.005 \\ 15 \pm 21 & 7 \pm 22 & 18 \pm 20 & 0.22\end{array}$
Per UI decrease
Per UI increase

Univariate logistic regression

Odds Ratio
(95\% confidence

(95\% confiden
interval)

P Valueł

$0.12(0.07-0.20)$

$1.29(1.05-1.58)$

$1.03(0.98-1.07)$

0.41

0.009

0.215

16 (55)

5 (62)

11 (52)

0.69

Yes vs. no

$0.70(0.08-4.55)$

0.69

12 (41)

1 (13)

11 (52)

0.09

Yes vs. no

$7.22(0.72-377.05)$

0.09

19 [10-54]

13 [11-15]

24 [7-56]

12 [5-23]

10 [6-13]

19 [5-36]

0.10

Per UI increase

Per Ul increase

$1.06(0.99-1.14)$

$1.07(0.98-1.16)$

0.017

$$
2 \text { [0-13] }
$$

1 [0-2]

10 [2-20]

0.05

Per UI increase

$1.06(0.99-1.15)$

0.051

0.019

$\begin{array}{lllllll}1.72 \pm 0.59 & 1.75 \pm 0.45 & 1.71 \pm 0.64 & 0.87 & \text { Per } 0.1 \text { UI decrease } & 0.09(0.02-0.36) & 0.87 \\ 1.26 \pm 0.42 & 1.62 \pm 0.34 & 1.12 \pm 0.37 & 0.002 & \text { Per } 0.1 \text { UI decrease } & 10.08(0.19-529.21) & 0.001 \\ & & & & & & \\ 1.38 \pm 0.52 & 1.47 \pm 0.55 & 1.34 \pm 0.45 & 0.08 & \text { Per } 0.1 \text { UI decrease } & 0.06(0.01-0.30) & 0.55 \\ 0.99 \pm 0.43 & 1.05 \pm 0.54 & 0.97 \pm 0.39 & 0.67 & \text { Per } 0.1 \text { UI decrease } & 0.06(0.01-0.45) & 0.66\end{array}$

Data are expressed as median [IQR], means \pm SD or counts (\%) when appropriate.

DWI denotes diffusion weighted imaging; FLAIR, fluid-attenuated inversion recovery and ${ }^{1} \mathrm{H}$-CSI, proton chemical shift Imaging.

† P-value for patients with a best numerical Cerebral Performance Categories 1 and 2 (good outcome) vs. those with categories 3 through 5 (poor outcome).

‡ P-value for Odds Ratio. 
Table 3. Area Under the Receiver Operating Characteristic Curve ( ROC $_{A}$ Ac) Values and Cutoff Values with at least $100 \%$ Specificity Predicting Unfavorable Outcome after

\section{Cardiac Arrest.}

\begin{tabular}{|c|c|c|c|c|c|}
\hline $\begin{array}{c}\text { ROC }_{\mathrm{AUC}} \\
\text { (95\% confidence } \\
\text { interval) }\end{array}$ & $\begin{array}{l}\text { Optimal } \\
\text { Cutoff }\end{array}$ & Specificity & Sensitivity & $\begin{array}{l}\text { Predictive } \\
\text { Positive } \\
\text { Value }\end{array}$ & $\begin{array}{c}\text { Negative } \\
\text { Predictive } \\
\text { Value }\end{array}$ \\
\hline & & \multicolumn{4}{|c|}{ Expressed in percent (95\% confidence interval) } \\
\hline $0.81(0.62-0.93)$ & $>7.25$ & $100(74-100)$ & $6(0-29)$ & $100(3-100)$ & $43(25-63)$ \\
\hline $0.84(0.66-0.95)$ & $\leq 4$ & $100(63-100)$ & $67(43-85)$ & $100(77-100)$ & $53(27-79)$ \\
\hline $0.79(0.60-0.92)$ & $>8$ & $100(63-100)$ & $57(34-78) \ddagger$ & $100(74-100)$ & $47(23-72)$ \\
\hline $0.88(0.71-0.97)$ & $\leq 1.32$ & $100(63-100)$ & $81(58-95)$ & $100(81-100)$ & $67(35-90)$ \\
\hline $0.85(0.71-0.99)$ & - & $100(63-100)$ & 76 (53-92) & $100(75-100)$ & $50(25-75)$ \\
\hline $0.86(0.73-0.99)$ & - & $100(63-100)$ & $62(38-82)$ & $100(79-100)$ & $62(32-86)$ \\
\hline $0.95(0.87-1.00)$ & - & $100(63-100)$ & 90 (70-99) & $100(82-100)$ & 80 (44-98) \\
\hline
\end{tabular}

DWI, diffusion-weighted imaging; FLAIR, fluid-attenuated inversion recovery; NAA/Cr, N-acetyl aspartate over creatine ratios and SAPS II, Simplified Acute Physiology Score II.

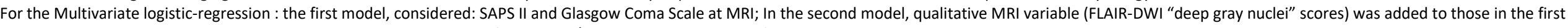
model. Finally, in the third model, predictive quantitative MRI variable (worst NAA/Cr ratio in Lenticular cores) was added to the second model.

† Sensitivity significantly different than the one of the NAA/Cr Lenticular cores $(\mathrm{P}<0.05)$. 